\title{
DGNSS-Vision Integration for Robust and Accurate Relative Spacecraft Navigation
}

\author{
V. Capuano ${ }^{1}$, A. Harvard ${ }^{1}$, Y. Lin ${ }^{1}$, and S.-J. Chung ${ }^{1}$ \\ ${ }^{1}$ Graduate Aerospace Laboratories, California Institute of Technology (GALCIT), Pasadena, \\ California, United States,vcapuano@caltech.edu, alexh@caltech.edu, yylin@caltech.edu, \\ sjchung@caltech.edu
}

\section{BIOGRAPHIES}

Vincenzo Capuano is Postdoctoral Researcher in the Aerospace Robotics and Control Lab at the California Institute of Technology, Pasadena, California. He received a PhD in Microsystems and Microelectronics at École Polytechnique Fédérale de Lausanne (EPFL), a Master of Engineering in Astronautic Engineering from the University of Rome "La Sapienza" and a Bachelor of Engineering in Aerospace Engineering from the University of Naples "Federico II". His current research activity focuses on Spacecraft Navigation, mainly relative navigation, vision-based, GNSS-based and multisensor-based.

Alexei Harvard is a Research Engineer in the Aerospace Robotics and Control Lab at the California Institute of Technology, Pasadena California. He received a B.S. in Mathematics from Caltech. His current research activity focuses on applying vision, sensor fusion, and machine learning to spacecraft navigation.

Yvette Lin is an undergraduate student at the California Institute of Technology.

Soon-Jo Chung received the S.M. degree in Aeronautics and Astronautics and the Sc.D. degree in Estimation and Control with a minor in Optics from MIT in 2002 and 2007, respectively. He received the B.S. degree in Aerospace Engineering from KAIST in 1998 (school class rank 1 out of 120). He is currently Bren Professor of Aerospace in the Graduate Aerospace Laboratories of the California Institute of Technology (GALCIT). Prof. Chung is also a Research Scientist of the Jet Propulsion Laboratory. For August 2009-August 2016, Prof. Chung was on the faculty of the University of Illinois at Urbana-Champaign. His research areas include nonlinear control and estimation theory and optimal/robust flight controls with application to aerial robotics, distributed spacecraft systems, and computer visionbased navigation. He is the recipient of the UIUC Engineering Dean's Award for Excellence in Research, the Beckman Faculty Fellowship of the U of Illinois Center for Advanced Study, the AFOSR Young Investigator Award, the NSF CAREER Award, and three best conference paper awards (2015 AIAA GNC, 2009 AIAA Infotech, 2008 IEEE EIT). Prof. Chung is an Associate Editor of the IEEE Transactions on Robotics and the AIAA Journal of Guidance, Control, and Dynamics.

\section{ABSTRACT}

Relative spacecraft navigation based on Global Navigation Satellite System (GNSS) has been already successfully performed in low earth orbit (LEO). Very high accuracy, of the order of the millimeter, has been achieved in postprocessing using carrier phase differential GNSS (CDGNSS) and recovering the integer number of wavelength (Ambiguity) between the GNSS transmitters and the receiver. However the performance achievable on-board, in real time, above LEO and the GNSS constellation would be significantly lower due to limited computational resources, weaker signals, and worse geometric dilution of precision (GDOP). At the same time, monocular vision provides lower accuracy than CDGNSS when there is significant spacecraft separation, and it becomes even lower for larger baselines 
and wider field of views (FOVs). In order to increase the robustness, continuity, and accuracy of a real-time on-board GNSS-based relative navigation solution in a GNSS degraded environment such as Geosynchronous and High Earth Orbits, we propose a novel navigation architecture based on a tight fusion of carrier phase GNSS observations and monocular vision-based measurements, which enables fast autonomous relative pose estimation of cooperative spacecraft also in case of high GDOP and low GNSS visibility, where the GNSS signals are degraded, weak, or cannot be tracked continuously.

In this paper we describe the architecture and implementation of a multi-sensor navigation solution and validate the proposed method in simulation. We use a dataset of images synthetically generated according to a chaser/target relative motion in Geostationary Earth Orbit (GEO) and realistic carrier phase and code-based GNSS observations simulated at the receiver position in the same orbits. We demonstrate that our fusion solution provides higher accuracy, higher robustness, and faster ambiguity resolution in case of degraded GNSS signal conditions, even when using high FOV cameras.

\section{INTRODUCTION}

Autonomous pose estimation of nearby orbiting objects is a key capability for space missions such as On-Orbit Servicing, On-Orbit Assembly, Active Debris Removal, Formation Flying, and the autonomous control of swarms of spacecraft. In most cases pose estimation must be directly performed in real time in order to enable autonomous relative navigation during close proximity operations, which are otherwise infeasible when based on ground-in-the-loop commands, due to communication delays and lack of coverage. Several technologies can be used to for autonomous pose estimation, depending on the operational environment, characteristics of the orbiting target, and on-board resources.

If the orbiting target spacecraft is actively cooperative, i.e. equipped with a Global Navigation Satellites System (GNSS) receiver and a communication link or a RF transmitting and receiving antenna, GNSS [32], [36], [29], [30] or RF based relative navigation can be a very accurate solution. It becomes possible to use millimeter accurate GNSS carrier-phase range observations instead of the standard meter accurate code-phase range observations to perform Carrier-phase Differential GNSS (CDGNSS), as done already using observations collected in some existing missions [44], [38]. Millimeter level accuracy has been achieved for relative positioning in Low Earth Orbit (LEO) missions by filtering GPS carrier phase measurements in post-processing with a model of the relative dynamics [44]. However, multipath (self-induced or induced by the other satellite), limited on-board computational resources, and imperfect knowledge of the spacecraft attitudes reduce the accuracy achievable on board in real time to the centimeter or decimeter level [22]. Indeed, the highest accuracy can be reached only in a fixed solution, which means only once the exact integer number of radio wavelengths between the GNSS transmitters and the receiver has been calculated [20], [42]. A low number of available GNSS satellites, low signal-to-noise-ratio (SNR), and poor receiver/transmitters relative geometry can prevent a fixed solution, only allowing a less accurate float solution. Therefore, the performance and robustness drastically decrease in higher earth orbits above the GNSS constellations when processing much weaker GNSS signals coming from the side lobes of the GNSS transmitters antennas, which results in significantly higher receiver noise and in signal outages [12], [13]. At the same time, monocular vision is another common technology for relative navigation [14]; however it typically provides lower accuracy than CDGNSS, particularly for larger baselines and wider field of views (FOVs).

In order to increase the robustness, continuity, and accuracy of a real-time on-board GNSS-based solution for relative spacecraft navigation, we propose a novel navigation architecture based on a tight fusion of carrier phase GNSS observations and monocular vision-based measurements that enables autonomous pose estimation of cooperative spacecraft in Low Earth Orbit (LEO) as well as in higher earth orbits with lower GNSS visibility.

Previous work has mainly focused on loosely coupling GNSS with vision [43], or deeply coupling the tracking modules of a GNSS receiver with a vision-aided inertial system, to reduce the receiver noise bandwidth by compensating for the user dynamics [8], [35]. In [34], it has been shown that one can couple vision with carrier-phase GNSS observations when utilizing a fixed base station to improve localization on earth and speed of ambiguity recovery. Unlike in other studies, in our architecture the vision-based observations are tightly fused with carrier-phase observations to provide accurate and robust real-time relative navigation between two non-stationary vehicles, both in an earth orbit.

We explore the robustness of our algorithm at different signal noise levels and satellite availability, as well as with different optical parameters. We show that our solution provides: 1) higher accuracy than if using GNSS or vision alone; 2) higher robustness than if using GNSS only or vision only (high accuracy also in case of GNSS signal 


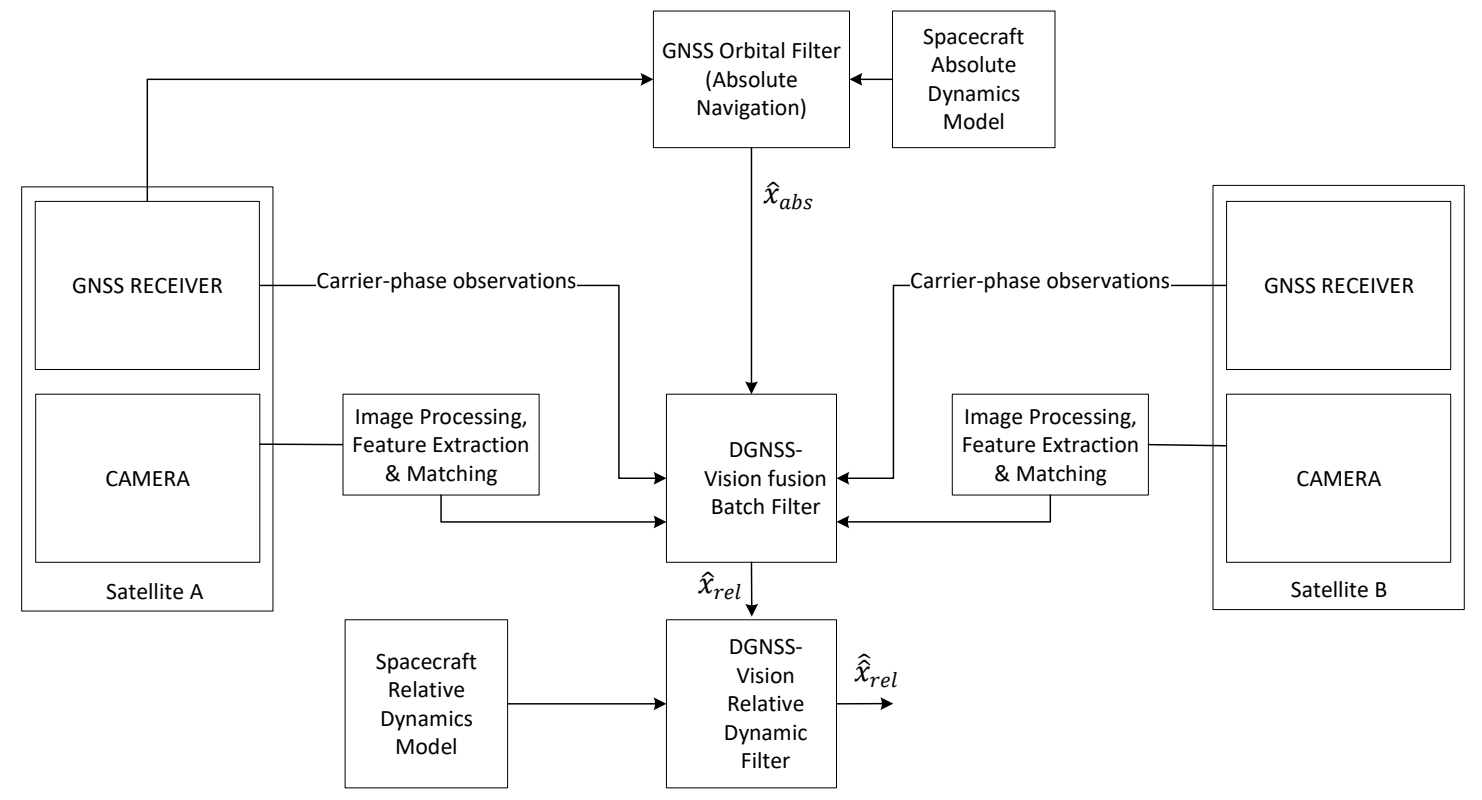

Figure 1: DGNSS-Vision integration architecture.

degradation and in case of larger baselines and larger FOVs); 3) successful GNSS ambiguity resolution also in case of degraded GNSS signal conditions, when it would not be possible with GNSS only; 4) GNSS ambiguity resolution drastically faster than with GNSS only, enabling real-time application.

The remainder of the paper is organized as follows. In Section 2, we describe the high level architecture and the implementation of the proposed multi-sensor navigation solution. In Section 3 we analyze and validate the proposed method, using a dataset of images synthetically generated according to a chaser/target relative motion in Geostationary Earth Orbit (GEO), along with realistic code-based and carrier-based GNSS observations simulated at the receiver position. Finally in Section 4, we draw the conclusion.

\section{ARCHITECTURE AND IMPLEMENTATION}

\subsection{High Level Architecture}

The complete architecture is shown in Fig. 1. Two satellites A and B, are assumed to be equipped with a GNSS receiver, a monocular camera, a star tracker, and a communication link. The GNSS receivers are pointed at the earth, while the cameras are pointed at each other, as much as possible. The satellites are also provided with LEDs for visual tracking. Both can receive GNSS signals and so can time-synchronize to flash the LEDs. The receiver to camera calibration is known. An on-board orbital filter based on GNSS code observations is used to estimate the absolute kinematic state $\hat{x}_{a b s}$ of satellite A. The absolute kinematic state estimate of satellite A is used as prior in a batch filter that estimates the relative kinematic state $\hat{x}_{\text {rel }}$ between satellite A and B, by fusing monocular images (of satellite A with the respect to satellite B and vice versa) with GNSS code and carrier phase observations (collected on board satellite A and B and shared between them using the RF communication link). The relative state estimate $\hat{x}_{\text {rel }}$ is fused with the predicted relative kinematic state obtained through the model of the relative orbital dynamics, in a sequential filter that provides a further refined relative state $\hat{\hat{x}}_{\text {rel }}$ estimate at higher output rates.

\subsection{Notation}

The following notation is assumed in the rest of the paper. 
- $P_{A}^{j, t}$ is the pseudo range measurement, from satellite $j$ to the receiver at $A$ at time $t$,

- $\rho_{A}^{j, t}$ is the true range from satellite $j$ to the receiver at $A$ at time $t$,

- $\phi_{A}^{j, t}$ is the tracked phase for satellite $j$ to the receiver at $A$,

- $x_{A}^{t}$ is the position of observer $A$,

- $R^{A}$ is the star tracker attitude estimate of $A$,

- $N_{A}^{i}$ is the integer ambiguity for satellite $i$ and the receiver at $A$,

- $p_{A}^{k}$ is the 3D position of LED $k$ of observer $A$, in its body frame,

- $\nabla \Delta(*)_{A B}^{j i, t}=\left(*_{A}^{j}-*_{A}^{i}\right)-\left(*_{B}^{j}-*_{B}^{i}\right)$ is the double difference of parameter $*$ at time $t$, relative to transmitters $i, j$ and receivers $A, B$,

- $T_{B}^{A}$ is the transformation from coordinate system $B$ to coordinate system $A$, considered as an element of $\mathbf{S E}(3)$.

We let $\mathscr{G}$ denote a GNSS receiver frame, $\mathscr{B}$ a center of mass (body) frame, and $\mathscr{C}$ a camera frame for each satellite. The relative transformations are denoted by $T_{\mathscr{R}, A}^{\mathscr{C}}$ and $T_{\mathscr{R}, B}^{\mathscr{C}}$. We will often shorten it to $T_{\mathscr{R}}^{\mathscr{C}}$ when it is understood we are working with a particular satellite. We will use $A$ and $B$ as shorthand for the frames $\mathscr{B}$ in $A$ and $B$ respectively.

The transformation from frame $A$ to $B$ (omitting the $t$ ) can be written as $T_{A}^{B}(p)=R_{A}^{B}(p)+x_{A}^{B}$, where $R_{A}^{B}=\left(R^{B}\right)^{-1} R^{A}$ and $x_{A}^{B}=\left(R^{B}\right)^{-1}\left(x_{A}-x_{B}\right)$. The relative position will be $x_{A}^{t}-x_{B}^{t}=\Delta x^{t}$.

\subsection{GNSS Receiver and Signals}

The main characteristics of the assumed receiver, relevant for the presented study, are summarized in Table 1. For this study, we assumed a receiver able to process signals down to $20 \mathrm{~dB}-\mathrm{Hz}$, as in GEO, this sensitivity is higher enough to track most of the available (and at least four) GPS L1 C/A signals [17]. This is not a strong assumption as a sensitivity of $15 \mathrm{~dB}-\mathrm{Hz}$ with the legacy GPS L1 C/A has been achieved in [9], with a spaecborne receiver specifically conceived for autonomous GNSS-based orbit determination above the GNSS constellation. We also assumed that the receiver has enough tracking channels to simultaneously track all available signals. For absolute navigation, when using code measurements, the receiver is assumed to process GPS signals with two different frequencies: L1 and L5. The processing of the L1 C/A signal is always assumed in signal acquisition (otherwise the primary code chipping rate of the L5 signal, being 10 times higher than the one of the L1 signal, would cause a significantly longer acquisition processing). Afterwards, once the L1 C/A is acquired, also GPS L5Q can be tracked, by exploiting the frequency relation between L1 and L5 and their code synchronization. The L5-band civilian signals are particularly advantageous, as their power is slightly higher than the L1 signals and they chipping rates is higher, yielding a lower tracking noise jitter for weak signal conditions in the ranging measurements. In addition, they have a pilot (data-free) channels that allows for longer integration time, enabling higher sensitivity of the receiver. At the same time, we also consider the L1-band, to process dual frequency iono-free combinations and for aiding the acquisition of the L5 frequency band signals. In relative navigation when using more precise carrier phase measurements and iono-free double differences, instead only the L1 frequency is used for the sake of simplicity.

The signal tracking configuration assumed for the receiver is reported in Table 2. In absolute navigation, in order to estimate the position and velocity of the chaser (satellite A) in the Earth Centered Inertial Frame (ECI), we assume to process code-based pseudorange observations and pseudorange rate observations derived from Doppler shift measurements of the receiver carrier frequency of both GPS L1 C/A and GPS L5 available signals. The relative position and velocity estimation between the chaser and the target (satellite B) are estimated by double differencing both code-based pseudorange observations and the more accurate ambiguous carrier phase-based observations of only GPS L1 C/A. 


\begin{tabular}{||cc||}
\hline Processed Signals: & GPS L1 C/A, GPS L5 \\
\hline $\begin{array}{c}\text { Acquisition and Tracking } \\
\text { Sensitivity: }\end{array}$ & $20 \mathrm{~dB}-\mathrm{Hz}$ \\
\hline $\begin{array}{c}\text { L1 C/A Acquisition } \\
\text { and Synchronization time: }\end{array}$ & $5 \mathrm{~min}$ \\
\hline Initial Clock Offset: & $10 \mathrm{~km}$ \\
\hline Initial Clock Drift: & $100 \mathrm{~m} / \mathrm{s}$ \\
\hline
\end{tabular}

Table 1: Assumed GNSS receiver characteristics.

\begin{tabular}{|c|c|c|c|}
\hline $\begin{array}{c}\text { Ionosphere } \\
\text { Crossing }\end{array}$ & $\begin{array}{l}\text { Navigation } \\
\text { Module }\end{array}$ & Signals & Observations \\
\hline Not & Absolute & GPS L5Q & Code-based \\
\hline Yes & Absolute & GPS L1 C/A and L5Q & $\begin{array}{c}\text { Code-based } \\
\text { Iono-free Combination }\end{array}$ \\
\hline Not & Relative & GPS L1 C/A & $\begin{array}{l}\text { Carrier Phase and Code } \\
\text { Double Differences }\end{array}$ \\
\hline Yes & Relative & GPS L1 C/A & $\begin{array}{l}\text { Carrier Phase and Code } \\
\text { Double Differences }\end{array}$ \\
\hline
\end{tabular}

Table 2: Tracking configurations.

\subsection{Monocular Image Processing, Feature Extraction and Matching}

Different pose determination approaches exist for pose determination based on monocular images, depending on whether the target is cooperative, uncooperative, known or unknown. The relative attitude and position (pose) of a Target Body Frame (TBF) with respect to the Camera Reference Frame (CRF) on board the chaser can be estimated using a number of features that are extracted from the acquired image. In our previous study [14] we focused on uncooperative targets, while here we assume a cooperative target, equipped with a set of artificial markers, i.e. LEDs and a RF transmitter. The artificial markers produce more distinguishable landmarks compared to natural features (e.g., corners) and their 3D position in TBF is already known since they are artificially placed on the target surface, according to specific known patterns. As a result, they can be easily detected in the acquired image.

In this study we assume a monocular camera on board both satellite A and B. In order to validate the proposed methods, as described in Section 3, different field of views (FOVs), different target geometries, and different illumination conditions were considered. In particular, we used two representative geometries, one of the Aura Spacecraft [1] and one of a $2 \mathrm{U}$ CubeSat [2]. According to these target geometries, camera parameters and a relative translational and rotational trajectory described in Section 3, synthetic images were generated using the tool Blender [3]. Figs. 2 and 3 are an example of respectively the Aura spacecraft seen from a camera with FOV $=32^{\circ}$ at $40 \mathrm{~m}$ distance and the $2 \mathrm{U}$ CubeSat seen from the same distance with FOV $=6^{\circ}$. For the majority of the data we focused on the CubeSat geometry with a fixed camera FOV of $90^{\circ}$ as it is possible to cover most of the possible views with a few of these cameras, which would allow constant visibility of the target.

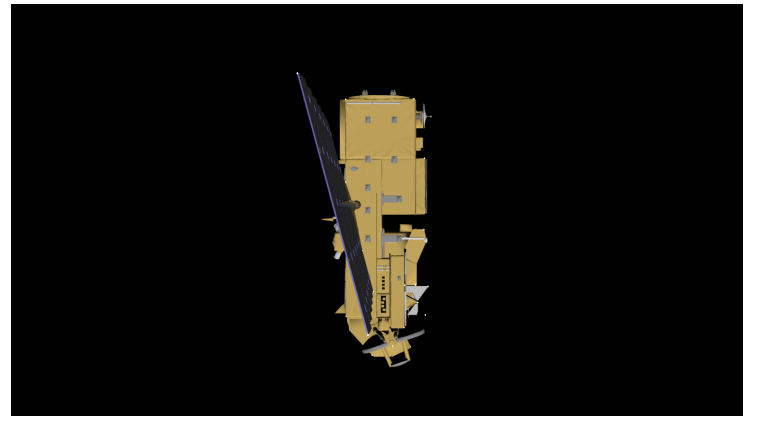

Figure 2: Aura spacecraft.

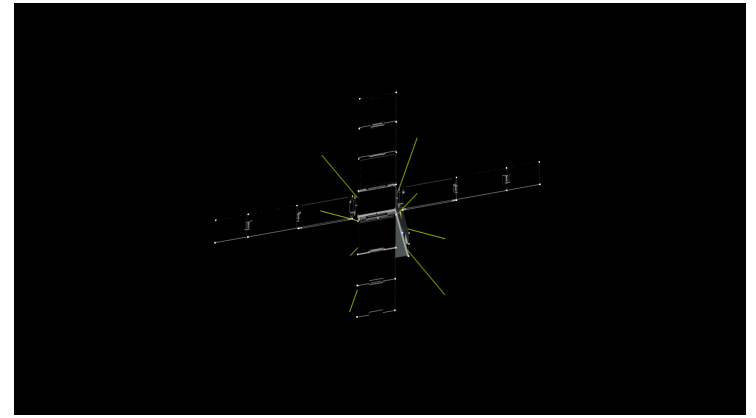

Figure 3: 2U CubeSat. 


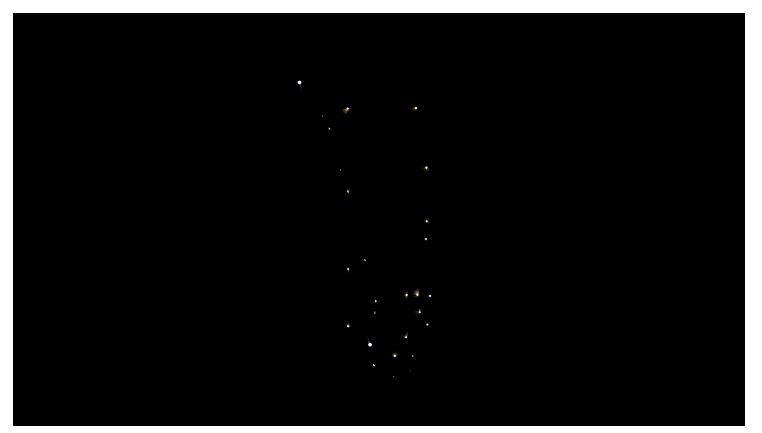

Figure 4: Feature extracted of the Aura spacecraft.

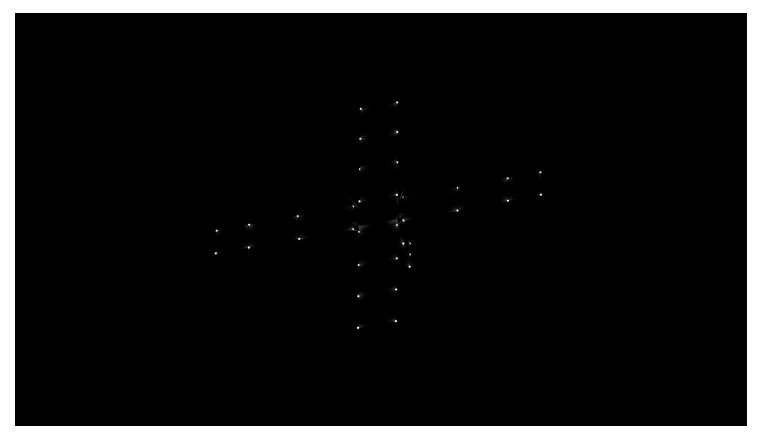

Figure 5: Feature extracted of the 2U CubeSat.

In a first step, the acquired monocular images are processed to extract the 2D coordinates of $n$ markers' centroid in the image frame. The clocks of the two satellites can be synchronized by their communication link and the GNSS receivers, so they can actively turn LEDs on and off in synchronization with the camera data capture. The satellite can capture an image with the LEDs on and then one with them off, allowing them to be subtracted to give a better contrast with the background (see Figs. 4 and 5). By utilizing the knowledge of the relative attitudes provided by the assumed star tracker measurements, the extracted points can be matched to the corresponding markers' $3 \mathrm{D}$ locations in the TBF, resulting in $n$ 2D-to-3D point correspondences. The problem of estimating the pose of a calibrated camera given a set of $\mathrm{n} 3 \mathrm{D}$ points in the world and their corresponding 2D projections in the image is known as Perspective- $n$-Point $(P n P)$. If among the $n$ 2D-to-3D point correspondences at least three are non-collinear in the TBF, the relative pose can be recovered [45]. Subsequent matching of extracted points to their 3D locations is done through optical tracking.

To solve the PnP problem, the correspondences between the extracted 2D points and the 3D locations of the LEDs that correspond to these points needs to be determined. Since the rotations of the chaser and the target are known within a small degree of error, and the camera intrinsics are known, we can determine the projections of the 3D LED locations onto the 2D image plane, up to scale and translation, with a small degree of error. Thus, the problem of establishing correspondences between the sets of extracted 2D points and of 3D LED locations is a problem of rigid point set registration with the addition of scaling and with a known rotation, between the sets of extracted 2D points and projected $2 \mathrm{D}$ points.

To solve the point set registration problem, the coherent point drift (CPD) algorithm [33] was used. To generate the initial set of 2D projections, an initial relative translation between the chaser and target can be obtained from the absolute navigation filters. Faster convergence will be achieved if the sets of initial projections and extracted points are closer. A simple method of choosing this initial relative translation is to assume the cameras of the chaser and target are pointed directly at each other, and that the distance between the chaser and target is such that a sufficient proportion (for example, $50 \%$ ) of estimated 2D projections lie within the bounds of most extreme extracted 2D points.

In the CPD rigid point set registration algorithm, the step in which the rotation matrix $R$ is updated was removed, fixing $R$ as its initialized value, the identity matrix, so that the scale $s$ and translation $t$ are the only transformations between the sets of estimated projections and extracted points for which we solve. After convergence, correspondences between the 2D extracted points and 3D LED locations are chosen by picking, for each extracted point, the LED whose projection has the highest probability of correspondence according to the probability matrix $P$ computed by the algorithm, given that this probability is sufficiently high (in this case, exceeds a threshold of $90 \%$ ). If no projection has a sufficiently large correspondence probability, the LED is assumed to not be visible in the image frame. In practice, this highest probability is very often either 0 or 1 , so the choice of whether to discard a correspondence is clear.

\subsection{DGNSS-Vision Fusion Batch Filter}

With respect to the high level architecture of Fig. 1, this filter provides the first estimate $\hat{x}_{\text {rel }}$ of the relative kinematic state between satellite A and B.

\subsubsection{Variables}

We run the batch over time points $t \in[k, l]$, solving for $\Delta x^{t}$ and $x_{A}^{t}$ at each second, along with the double differenced carrier phase integer ambiguities $\nabla \Delta(N)_{A B}^{j 1}$ as long as satellite 1 and $j$ maintain phase lock. 
If phase lock is lost and restablished then a new constant integer ambiguity is calculated and treated as a new variable. In the case that the base satellite (here satellite '1') loses lock, we can characterize subsequent double difference measurements by $\nabla \Delta N_{A B}^{j i}=\nabla \Delta N_{A B}^{j 1}-\nabla \Delta N_{A B}^{i 1}$ when using satellite $i$ as a base.

\subsubsection{Measurements}

The ranges correspond to $\rho_{A}^{j}$ as $\left\|x_{j}^{t}-T_{\mathscr{B}, A}^{\mathscr{G}} x_{A}^{t}\right\|$, and similarly for $\rho_{B}^{j}$. Then the double differenced phase measurements are characterized by

$$
\lambda_{L 1} \nabla \Delta(\phi)_{A B}^{j 1}=\nabla \Delta(\rho)_{A B}^{j 1}+\lambda_{L 1} \nabla \Delta(N)_{A B}^{j 1}+\nabla \Delta(w)_{A B}^{j 1} .
$$

We define $\mathrm{w}_{A}^{i, t}$ as the double differenced carrier phase measurement error. The measurement will then have a covariance matrix of $\sigma_{\phi}(2 \mathbb{I}+2 \mathbb{J})$ where $\frac{\sigma_{\phi}}{\lambda_{L 1}}$ is the estimated phase measurement standard deviation, $\mathbb{I}$ is the $(J-1) \times(J-1)$ identity matrix, and $\mathbb{J}$ is the $(J-1) \times(J-1)$ unit matrix.

Let $P_{B}$ be the projection operator for B's linear camera, and the observation of LED $k$ of $A$ at time $t$ in $B$ 's camera be $o_{B}^{k}$. This is characterized by

$$
o_{B}^{k, t}=P_{B}\left(T_{A}^{B, t}\left(p_{A}^{k}\right)\right)+\varepsilon_{B}^{k, t} .
$$

$\varepsilon_{B}^{k, t}$ is the point image localization error assumed to be $\mathscr{N}\left(0, \sigma_{o}^{2}\right)$. We do a similar measurement for $o_{A}^{k, t}$. The observations are obtained by using the known geometry of the satellite LED and the shared star-tracker pose.

We also create an extra constraint on the relative positions by utilizing the code-based pseudorange measurements as

$$
\nabla \Delta(P)_{A B}^{j 1}=\nabla \Delta(\rho)_{A B}^{j 1}+\nabla \Delta(v)_{A B}^{j 1},
$$

For each variable we can incorporate priors such as $\widehat{x_{A}^{t}}$ from the absolute filter or $\widehat{\Delta x^{t}}$ propagated from the relative filter. We can also propagate the floating solutions for $\nabla \Delta(N)_{A B}^{j i}$ as $\widehat{\nabla \Delta N_{A B}^{j i}}$, along with their resulting marginal covariances from previous solutions.

\subsubsection{Factor Graph Formulation}

To incorporate multiple measurement types and test different conditions, we propose to structure the filter as a factor graph, as is similar to what is done for SLAM implementations [26]. Figure 6 shows an example formulation, which captures the variable relationships and sparse structure. Assuming the noise is Gaussian we can apply graph ordering techniques to have a more efficient representation of the underlying data when doing cholesky factorization [21] for when we solve the locally linearized problem using sparse linear algebra.

\subsubsection{Iterative Solution}

To solve for the MAP (maximum a posteriori) estimate of $\Delta x^{l}$, we initially linearize the least square problem obtained from the factor graph, similar to what is done in $[32,34]$. This gives us a sparse linear equation of the form

$$
A \Delta \mathbf{y}=b,
$$

where $A$ is determined by the Jacobian and covariances of our measurements and $b$ by the residuals and covariances. $\Delta \mathbf{y}$ is the linear correction to our state

$$
\mathbf{y}=\left[\begin{array}{c}
x_{A}^{t} \\
\Delta x^{t} \\
\nabla \Delta(N)_{A B}^{j 1}
\end{array}\right] .
$$

This is approximated as a linear regression problem with normal noise on $b$. The least square estimate is obtained as

$$
\widetilde{\Delta y}=\left(A^{\top} A\right)^{-1} A^{\top} b,
$$

with $Q=\left(A^{\top} A\right)^{-1}$ being the covariance of the solution. As discussed above, we can use the graph representation to get an efficient sparse ordering of $A$ under the constraint that the double-differenced integer ambiguities $\nabla \Delta(N)_{A B}^{j 1}$ are the last terms, that gives us an efficient way to do the cholesky factorization $R^{\top} R=A$ to solve for the float solution 


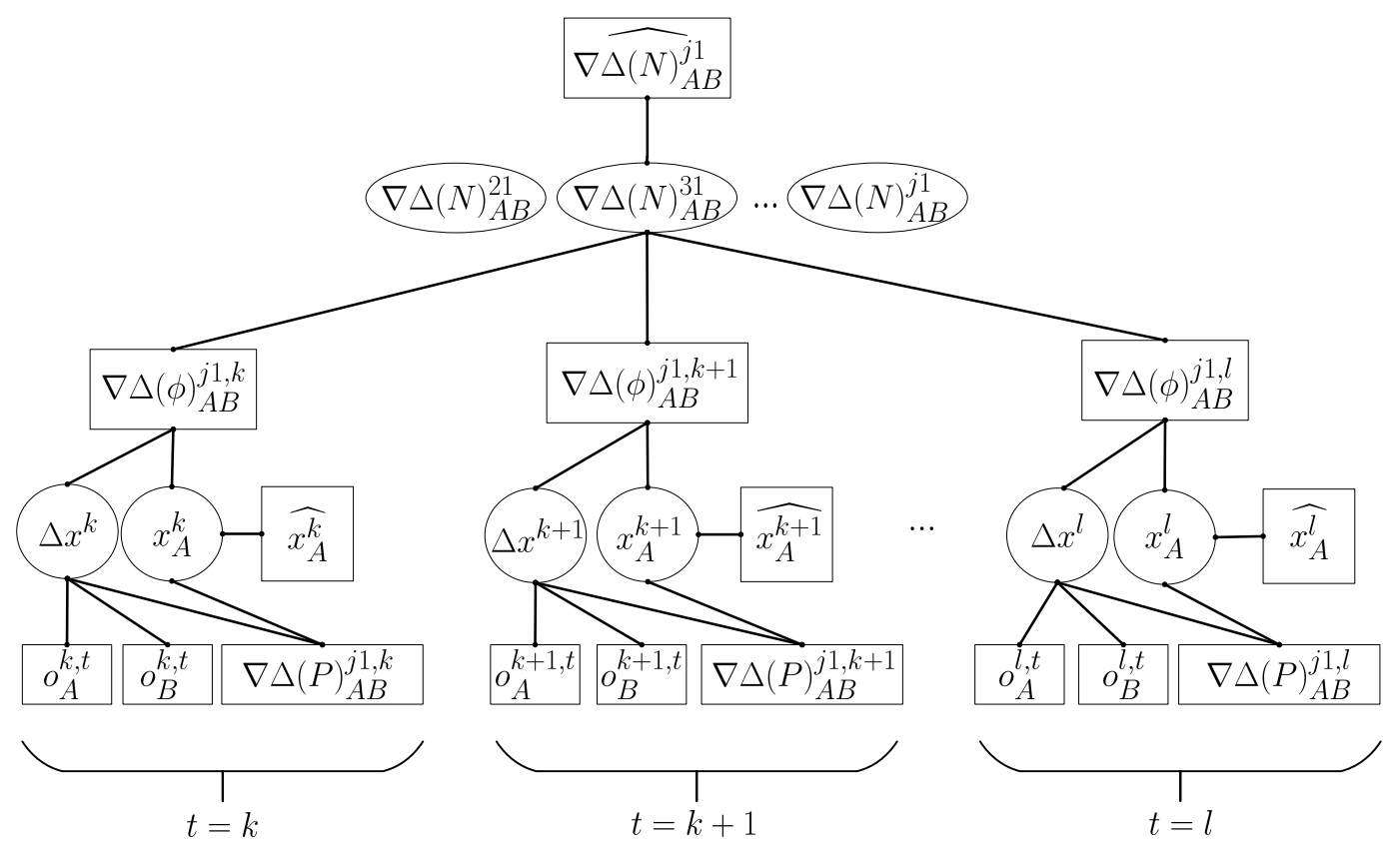

Figure 6: Batch filter as a factor graph.

$\widetilde{\Delta y}$. Then using $R$ we can obtain $Q_{N}$, the covariance of the float $\overline{\nabla \Delta(N)_{A B}^{j 1}}$ terms, and use the MLAMBDA method [19] to solve

$$
\min _{\nabla \Delta(N)_{A B}^{j 1} \in \mathbb{Z}^{J-1}}\left(\overline{\nabla \Delta(N)_{A B}^{j 1}}-\nabla \Delta(N)_{A B}^{j 1}\right)^{\top} Q_{N}^{-1}\left(\overline{\nabla \Delta(N)_{A B}^{j 1}}-\nabla \Delta(N)_{A B}^{j 1}\right) .
$$

After we solve for $\nabla \Delta(N)_{A B}^{j 1}$, we can correct and then fix the integer terms and then solve the cost function by doing a standard iterated nonlinear least squares solution for our factor graph. After convergence a new set of double differenced integer ambiguity corrections is computed in the same way, with the algorithm terminating once the ambiguities do not change. We can then use the covariance $Q_{N}$ along with $\nabla \Delta(N)_{A B}^{j 1}$ as the prior $\widehat{\nabla \Delta(N)_{A B}^{j 1}}$ for subsequent batches.

\subsection{GNSS-based Orbital Filter for Absolute Navigation}

The adaptive orbital filter developed in [16] was adopted to provide an accurate absolute state estimate $\hat{x}_{a b s}$, which includes chaser position vector $r_{a b s}$ and velocity vector $v_{a b s}$ in the ECI frame, as well as the receiver clock bias $d \rho_{t}$ and drift $d \dot{\rho}_{t}$. The filter processes directly a vector of the available GNSS code-based pseudoranges $P$ and a vector of their corresponding pseudorange rates $\dot{P}$. It is based on a reduced dynamic approach, which augments the state vector with empirical accelerations $a_{e}$, in order to compensate for mismodeling errors of the spacecraft dynamics. The state vector and the measurement vector are defined as follows.

$$
\begin{aligned}
& x_{a b s}=\left[r_{a b s}, v_{a b s}, d \rho_{t}, d \dot{\rho_{t}}, a_{e}\right]^{\top}, \\
& z_{a b s}=[P, \dot{P}]^{\top}
\end{aligned}
$$

In this implementation, an Extended Kalman Filter (EKF) was used to fuse the GNSS observations $z_{a b s}$ and their prediction $h\left(\hat{x}_{a b s}\right)$, obtained propagating the last estimate $\hat{x}_{a b s}$. Spacecraft's absolute position and velocity vectors in the ECI are predicted by integrating the dynamics of the spacecraft:

$$
a(r, t)=a_{g}(r, t)+a_{s}(r, t)+a_{m}(r, t)+a_{s r p}(r, t)+a_{r}(r, t)
$$

where $r$ is the position vector and $t$ is dynamical time, $a$ is the total acceleration of the spacecraft, $a_{g}$ the acceleration due to Earth's gravity, $a_{s}$ the acceleration due to the Sun, $a_{m}$ the acceleration due to the Moon and $a_{s r p}$ the one due to the 
solar radiation pressure. A complete description of each orbital force implementation can be found in [10]. In addition, in the reduced-dynamic implementation, the pseudo-stochastic parameters $a_{e}$ representing the empirical accelerations, are modelled as first order stationary Gauss-Markov process, therefore exponentially correlated. The filter makes use of an adaptive tuning of the covariance matrix of the measurements, as function of the pseudorange and pseudorange rate predicted errors, function of the carrier-to-noise-ratio of the processed signals at the receiver position [16]. Being the GNSS observations and their dynamics-based prediction characterized by dissimilar error characteristics, their fusion can provide a position and velocity estimation more accurate than the one achievable individually. The filter was tuned and adapted to be used in GEO, processing GPS L1 and L5 dual frequency observations. A complete description of the orbital filter architecture and implementation is provided in the previous studies [16], [11] and [10].

\subsection{Relative Navigation Filter}

A relative navigation filter that makes use of the relative translational orbital dynamics between chaser and target [46] was implemented and used to further process the relative position estimate of the batch filter $\hat{x}_{\text {rel }}$ and to provide a refined estimate $\hat{\hat{x}}_{r e l}$ with higher output rate. In this study, as in [15], we adopted a special form of the Kalman filter, the H-infinity or minimax filter [41], based on the H-infinity optimal estimation that guarantees the smallest estimation energy error for all possible disturbances of the fixed energy. Such a filter minimizes the worst case estimation error without any assumptions on the probability distribution of the system and measurement noise and therefore can better handle modelling errors and noise uncertainty, resulting more robust when processing optical measurements in presence of variable illumination conditions. As consequence, it outperforms the standard Kalman Filter [27], which instead guarantees an optimal estimation by minimizing the expected variance of the estimation error under the assumption that system and measurement noise have white Gaussian distribution. In general, a linear model of the system and of the measurements, can be represented as follows.

$$
\begin{aligned}
x_{k+1} & =\Phi_{k} x_{k}+w_{k}, \\
z_{k} & =H_{k} x_{k}+v_{k}
\end{aligned}
$$

where the subscript $k$ indicates the $k$-th time step, $x_{k}, z_{k}, \Phi_{k-1}, H_{k}, w_{k}$ and $v_{k}$ are respectively the state vector, the measurement vector, the transition matrix, the measurement matrix, the system noise and the measurement noise, random with possibly unknown statistics or deterministic, potentially nonzero mean.

According to [41], the following formulation can be used for the discrete time prediction and update filtering steps.

$$
\begin{aligned}
K_{k} & =P_{k}\left[I-\theta P_{k}+H_{k}^{\top} R_{k}^{-1} H_{k} P_{k}\right]^{-1} H_{k}^{\top} R_{k}^{-1}, \\
\hat{x}_{k+1} & =\Phi_{k} \hat{x}_{k}+\Phi_{k} K_{k}\left(z_{k}-H_{k} \hat{x}_{k}\right), \\
P_{k+1} & =\Phi_{k} P_{k}\left[I-\theta P_{k}+H_{k}^{\top} R_{k}^{-1} H_{k} P_{k}\right]^{-1} \Phi_{k}^{\top}+Q_{k},
\end{aligned}
$$

where $K_{k}$ is the filter gain, $P_{k}$ is the covariance of the filter, $\theta$ is a performance bound specified by the user, while $Q_{k}$ and $R_{k}$ are the covariance matrices respectively associated with $w_{k}$ and $v_{k}$, symmetric. In our case, the state vector and the measurement vector are defined as follows.

$$
x_{r e l}=\left[r_{r_{e l}}, v_{r_{e l}}\right]^{\top}, \quad z_{r e l}=\left[\hat{r}_{r_{e l}}, \hat{v}_{r_{e l}}\right]^{\top}
$$

where $\hat{r}_{r_{e l}}$ and $\hat{v}_{r_{e l}}$ are respectively the relative position and velocity estimates of the batch filter described in Section 2.5 .

\section{SIMULATED PERFORMANCES}

The proposed methods were validated in simulation. We used a dataset of images synthetically generated according to a chaser/target relative motion in Geostationary Earth Orbit (GEO), and realistic carrier phase and code GNSS observations simulated at the receiver position in the same orbit, taking into account all different GNSS signal delay sources as well as their availability based on their carrier-to-noise-ratio and receiver sensitivity. 


\begin{tabular}{||cc||}
\hline Semi Major Axis: & $42167.043348 \mathrm{~km}$ \\
\hline Eccentricity: & 0.000289 \\
Inclination: & $0.081 \mathrm{deg}$ \\
\hline Argument of Perigee: & $354.152 \mathrm{deg}$ \\
Right Ascension of & \\
the Ascending Node (RAAN): & $68.784 \mathrm{deg}$ \\
\hline Mean Anomaly: & $240.837 \mathrm{deg}$ \\
\hline
\end{tabular}

Table 3: Initial Keplerian orbital parameters of the considered chaser orbit.

\begin{tabular}{||cc||}
\hline Target initial separation: & $15 \mathrm{~m}$ along chaser velocity axis \\
(Position of TBF in LVLH frame) & (y axis of the LVLH frame) \\
\hline Target initial relative rotation rate: & $1 \mathrm{deg} / \mathrm{s}$ around one principal axis of the TBF \\
(Rotation rate of TBF in LVLH frame) & $\left.\left(\begin{array}{lll}0 & 1 & 0\end{array}\right] \mathrm{deg} / \mathrm{s}\right)$ \\
\hline Chaser camera boresight direction: & aligned with chaser velocity axis \\
& $\left.\left(\begin{array}{lll}0 & 1 & 0\end{array}\right]\right)$ \\
\hline
\end{tabular}

Table 4: Initial relative kinematics.

\subsection{Simulation Models}

\subsubsection{Absolute Kinematics and Dynamics}

The orbit assumed for the chaser (satellite A) was the one of the Intelsat 904 (IS-904) communication satellite (see Fig. 7), launched on February 23rd 2002 and still active [4]. The initial Keplerian orbital parameters at time 00:00 of May 26th 2016 are reported in the Table 3, downloaded from the Standard Object Data Service of AGI's (Analytical Graphics INC) library. Then, the motion of the receiver was propagated by the precise STK HPOP propagator [5] from the initial condition as function of the perturbing accelerations (such as the full Earth gravitational field, third-body gravity, atmospheric drag and solar radiation pressure). The receiver antenna was assumed to be Earth pointing.

\subsubsection{Relative Kinematics and Dynamics}

The assumed relative transnational trajectory of the target (satellite B) with respect to the chaser (satellite A) is identified by the initial conditions reported in Table 4 and propagated by integrating the nonlinear dynamics model presented in [24], which describes the eccentric trajectory of the target in the LVLH (Local Vertical Local Horizontal) reference frame of the chaser. The relative rotational dynamics is propagated as suggested in [39], combining the Euler equations for both chaser and target.

\subsubsection{GNSS Constellation, Signals and Observations}

The GPS constellation was modelled according to the almanac of the 1st July 2005 that includes a total of 29 satellites (PRN from 1 to 31 except 12 and 17)), as illustrated in Fig. 7.

Taking into account gain patterns of both transmitter and receiver antennas and free space signal propagation losses, the signal power levels received at the receiver's antenna position were modelled as in equation (1) of the previous study [12], according to the guaranteed minimum received signal power (see [7] and [6]) for both GPS signals considered. 3D receiver and transmitters' antenna patterns for each satellite and for each frequency of the constellation were used. Figure 8 illustrates the ones for the GPS L1 block IIF for example. Note that each curve represents the gain vs elevation for a different azimuth. The gain is normalized to $0 \mathrm{~dB}$ at boresight (located at $0 \mathrm{deg}$ elevation). More details about the assumed transmitters' antenna patterns can be found in [40]. Figure 9 shows the antenna pattern assumed for the receiver, designed for the reception of GNSS signals at GEO in [31] to increase the gain for only the signals coming from the transmitter's side lobes. 


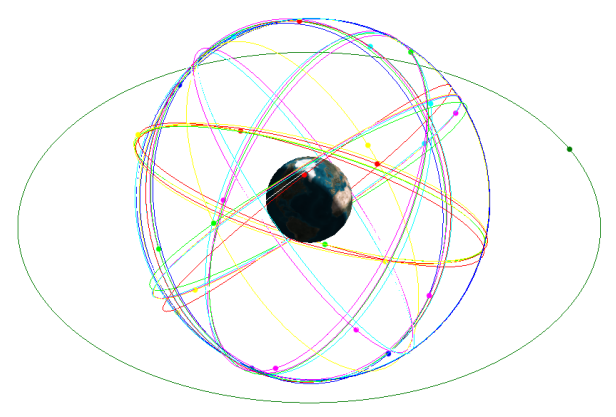

Figure 7: Chaser orbit and GPS constellation.

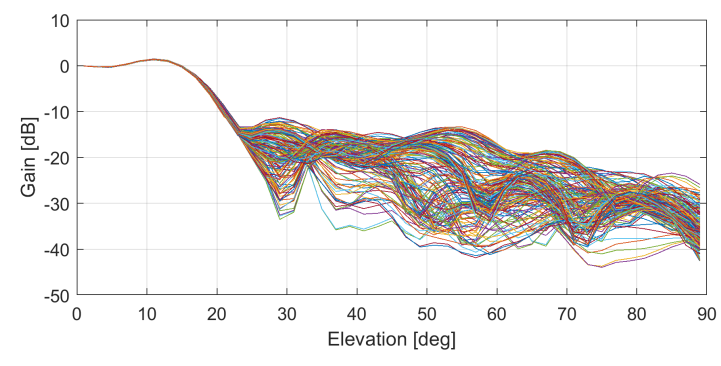

Figure 8: Transmitters' antenna patterns.

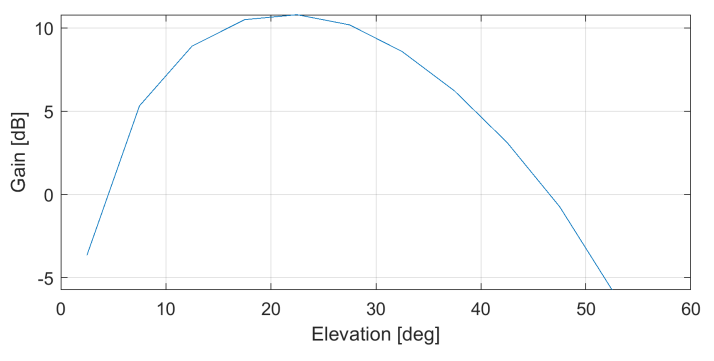

Figure 9: Receiver antenna pattern.

Note that although only the GPS constellation and signals are considered in this study, the results are extendible also to the case of other constellations, or to the case of combined constellations for which better availability and thus better Geometric Dilution Of Precision (GDOP) and performance can be reached.

The code-based GNSS pseudorange observations were modelled according to the previous study [18], accounting for transmitters' clock error and broadcast ephemeris error, atmospheric delay, multipath effect and receiver errors. The specific strategy (described in [18]) was adopted here to deal with possible ionospheric delays. When only one frequency is available (this is possible since different antenna patterns are used for L1 and for L5), all the signals that cross the ionosphere (and the troposphere as well below) are simply discarded. Instead, when two frequencies $f_{m} 1$ and $f_{m} 2$ from the same GNSS satellite are processed at the receiver position providing two measurements $m 1$ and $m 2$, we also process the signals crossing the ionosphere, assuming a ionosphere-free combination of the two measurements as follows.

$$
\sigma_{i f}^{2}=\frac{1}{\left(\omega_{m 1, m 2}-1\right)^{2}}\left[\omega_{m 1, m 2}^{2} \sigma_{m 1}^{2}+\sigma_{m 2}^{2}\right]
$$

where $\omega_{m 1, m 2}^{2}=\frac{f_{m}^{2} 1}{f_{m}^{2} 2}$, for $f_{m 1}>f_{m 2}$ and $\sigma_{m}^{2}$ is the variance for the frquency $f_{m}$. We considered a standard deviation value of $0.5 \mathrm{~m}$ for transmitter's clock and broadcast ephemeris errors [23], a standard deviation of $0.2 \mathrm{~m}$ for possible multipath [28]. As done in [17], the receiver error was modeled as function of the receiver characteristics and of the carrier-to-noise-ratio $C / N_{0}$ to account for the large variations of the signals power level at the receiver position above the GPS constellation (see Eq. (2:4) of [17]).

The pseudorange rates were modelled from the Doppler shift measurements of the received carrier frequency, by simply multiplying the Doppler shift observation with the wavelength of the carrier. The Doppler tracking jitter was assumed as the main source of error in Doppler frequency estimation, modelled as function of the power level at the receiver position (see Eq. (2:7) of [17]).

The carrier-phase observations for GPS L1 C/A were modelled according to [37], taking into account multipath, antenna phases-center variation and thermal noise as function of the receiver characteristics and signals power level at the receiver position (see Eq. (9) of [37]). In the GEO scenario considered in this study, the maximum carrier phase measurement noise is $0.0115 \mathrm{~m}$. However, in higher orbits, as in a earth to moon transfer orbits, even larger thermal noise would be experienced due to the processing of weaker signals. In addition higher multipath (here assumed of 
$0.005 \mathrm{~m}$ [37]) self-induced or induced by other spacecraft could be experienced in space [25].

\subsection{Test Cases}

Different tests were performed to validate the robustness in ambiguity resolution of the proposed DGNSS-Vision fusion (described in Section 2.5) and the robustness and accuracy of the same solution $\hat{x}_{\text {rel }}$, as well as of the solution $\hat{\hat{x}}_{\text {rel }}$ refined by means of the dynamics filter (described in Section 2.7), in relative position and velocity estimation.

In addition, we compared the GNSS/Vision integrated relative navigation solutions $\hat{x}_{\text {rel }}$ and $\hat{\hat{x}}_{\text {rel }}$ to the one achievable when using only GNSS and only Vision. Then we evaluated them also in case of reduced GNSS availability (when only a limited number of GNSS observations are available) and for different FOVs. The same tests were performed for both target geometries of Figs. 2 and 3. In this study we will report the results obtained for the CubeSat of Fig. 3.

\subsection{Results}

Table 5 shows the results from trying to recover the integer ambiguity in a single time step with the method described in Section 2.5, assuming only 5 available satellites while varying the underlying carrier phase noise $\sigma_{\phi}$ from the minimum value simulated for this scenario to its maximum $(0.0115 \mathrm{~m})$ and to values four times higher.

Table 6 shows how likely it is to recover the integer ambiguity over a given time span (a batch size of 4 corresponds to 4 seconds) and satellite count, assuming the phase noise is $0.0115 \mathrm{~m}$. The GNSS-Vision fusion algorithm was able to very quickly recover the ambiguity in situations where conventionally GNSS is extremely slow, particularly when the phase tracked satellite count is very low.

Table 7 analyzes the effects of different fields of view of the camera. In this simulation, it was assumed that the camera could see at most 9 LEDs at any given time point, to compensate for their reduced visibility at larger FOVs. The vision based points measurements were also given a conservative standard deviation of 1 pixel, even though standard localization algorithms are usually subpixel in accuracy. The standard deviations are from the unfiltered position estimates while using the phase noise of $0.0115 \mathrm{~m}$. We note that the GNSS-vision result is not affected very much by an increasing FOV despite the recovered vision solution becoming much worse. This is explained by the error covariances of the GNSS and Vision solutions being complementary due to the Relative Passive Orbit, where the optical axis is approximately perpendicular to the radial vector to the target which is the dominating error component of GNSS, as shown in Fig. 10.

Table 8 compares the mean recovery times between a GNSS only and GNSS-vision system under different satellite counts and carrier phase noises, when the receivers maintains a PLL lock on the carrier signal and the priors on the floating double difference ambiguity errors are propagated between batches. Recovery times for low noise and high satellite counts are similar to what is achieved in [32], where unique resolution happens within about 3 seconds. The authors expect that for larger GDOP at higher altitudes the recovery times for the GNSS only systems will be much slower.

Figure 11 shows the norm of the error of the absolute state (position and velocity) estimation vector $\hat{x}_{a b s}$. Thanks to the assumed high receiver sensitivity, an average of 25 GPS satellites are available over time with an average GDOP of 4.5. An accuracy better than $1 \mathrm{~m}$ and of $1 \mathrm{~mm} / \mathrm{s}$ in absolute position and absolute velocity estimation is achieved adopting the dynamics filter described in Section 2.6. Figs. 12, 13 and 14 show the error norm of the relative state estimates $\hat{x}_{\text {rel }}$ and $\hat{\hat{x}}_{\text {rel }}$, respectively when fusing GNSS with vision, when using only GNSS and when using only monocular vision. Clearly the GNSS-Vision integrated solution with a std of the error smaller than 2 mm, outperforms the one obtained with vision and GNSS individually, with a std of the error of respectively $2.5 \mathrm{~cm}$ and $5 \mathrm{~mm}$. In Figs. 15 and 16 we can see what is the effect of a reduced GNSS signal availability. Figure 15 shows the absolute state estimation error (in position and velocity), when, starting from the 5500th second only 3 and 2 GPS observations are used, instead of all the available ones. As expected, when less than four observations are available the filter estimation error starts to slowly diverge. Figure 16 shows what is the effect in the relative state estimation; in the time window between the 5500th and 6000th seconds, neither the estimate of the GNSS-Vision batch filter $\hat{x}_{\text {rel }}$ nor the refined estimate $\hat{\hat{x}}_{\text {rel }}$ are affected by the drift in absolute estimation and by the reduced number of available GPS observations. Following the sudden drop of GPS observations from 25 to 3 and from 25 to 2 at the 5500th second, the GNSS-Vision batch filter estimates become noisier, however the dynamics filter is able to robustly limit the loss of accuracy from $1.8 \mathrm{~mm}$ to 2.2 and $2.5 \mathrm{~mm}$ respectively for 3 and 2 only available GPS observations. 


\begin{tabular}{||c|cccccccccccc||}
\hline$\sigma_{\phi}(\mathrm{m}):$ & 0.001 & 0.003 & 0.005 & 0.0075 & 0.01 & 0.0125 & 0.015 & 0.02 & 0.025 & 0.03 & 0.035 & 0.04 \\
\hline GNSS only (\%): & 3.3 & 1.0 & 0.03 & 0 & 0.1 & 0.1 & 0.1 & 0.1 & 0 & 0 & 0 & 0 \\
GNSS-vision (\%): & 100.0 & 100.0 & 99.9 & 99.2 & 96.7 & 89.5 & 81.4 & 63.1 & 54.1 & 49.6 & 48.2 & 46.7 \\
\hline
\end{tabular}

Table 5: $\%$ of ambiguities recovered from a single batch filter step, 5 satellites, FOV $=90^{\circ}$

\begin{tabular}{|c|c|c|c|c|c|c|c|c|c|}
\hline \multicolumn{2}{|c|}{ Satellites (\#): } & 4 & 5 & 6 & 10 & 15 & 20 & 25 & All \\
\hline \multirow[t]{5}{*}{ GNSS only (\%): } & Batch size 1 & 0.0 & 0.0 & 0.2 & 4.2 & 38.1 & 87.4 & 98.0 & 98.0 \\
\hline & Batch size 2 & 0.0 & 0.0 & 0.4 & 21.4 & 90.8 & 99.8 & 100.0 & 100.0 \\
\hline & Batch size 3 & 0.0 & 0.4 & 1.0 & 45.3 & 98.4 & 100.0 & 100.0 & 100.0 \\
\hline & Batch size 4 & 0.0 & 0.4 & 1.2 & 64.9 & 99.8 & 99.8 & 100.0 & 100.0 \\
\hline & Batch size 1 & 83.8 & 88.2 & 90.6 & 99.8 & 100.0 & 100.0 & 100.0 & 100.0 \\
\hline \multirow{2}{*}{ GNSS-vision (\%): } & Batch size 2 & 98.4 & 99.4 & 99.6 & 100.0 & 100.0 & 100.0 & 100.0 & 100.0 \\
\hline & Batch size 3 & 100 & 100.0 & 100.0 & 100.0 & 100.0 & 100.0 & 100.0 & 100.0 \\
\hline
\end{tabular}

Table 6: $\%$ of ambiguities recovered from a single time step batch $\sigma_{\phi}=0.0115, \mathrm{FOV}=90^{\circ}$

\section{CONCLUSION AND FUTURE WORK}

Although CDGNSS positioning is more accurate than a code-based DGNSS, it is characterized by a longer convergence time and lower robustness, particularly in degraded environments such as in GEO and in higher earth orbits,

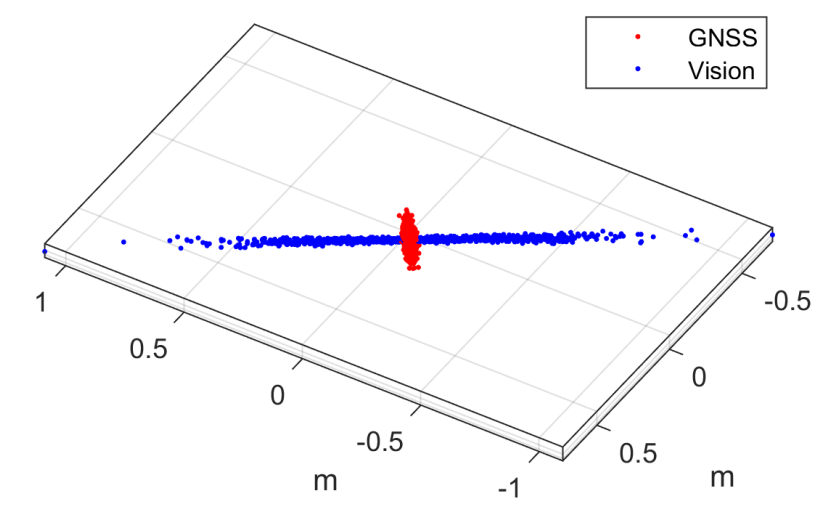

Figure 10: GNSS and Vision error ellipsoids, $\mathrm{FOV}=90^{\circ}, \sigma_{\phi}=0.0115$.
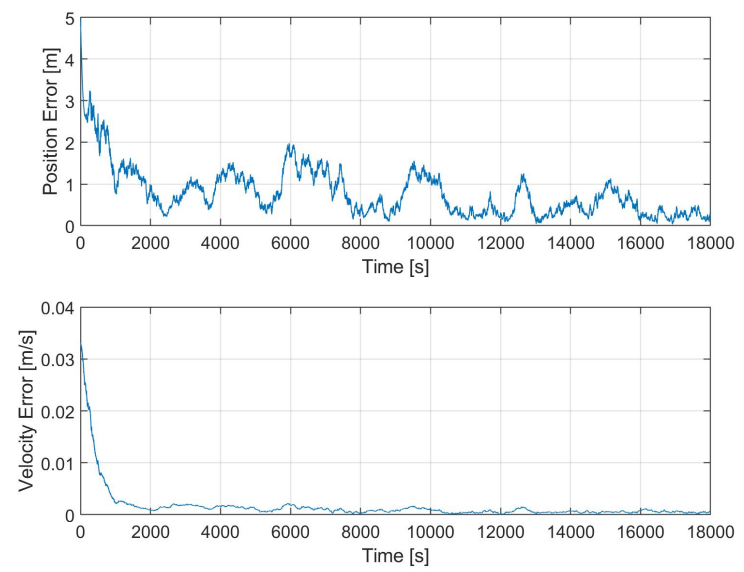

Figure 11: GNSS absolute estimation error.
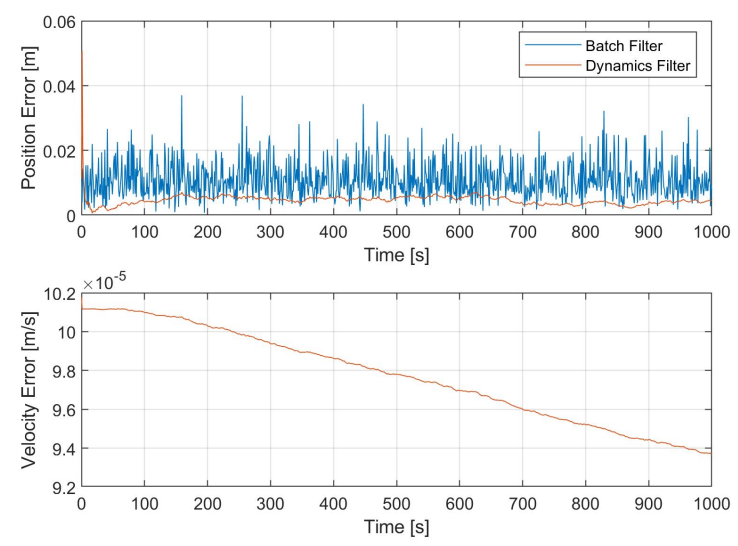

Figure 12: DGNSS-Vision error. 


\begin{tabular}{||cc|cccccccc||}
\hline Satellites (\#): & & 4 & 5 & 6 & 10 & 15 & 20 & 25 & All \\
\hline GNSS only (m): & & - & - & - & 0.047 & 0.033 & 0.027 & 0.026 & 0.026 \\
GNSS-vision (m): & $6^{\circ}$ & 0.010 & 0.008 & 0.008 & 0.007 & 0.007 & 0.006 & 0.006 & 0.005 \\
& $10^{\circ}$ & 0.010 & 0.010 & 0.010 & 0.008 & 0.008 & 0.007 & 0.006 & 0.006 \\
& $20^{\circ}$ & 0.014 & 0.012 & 0.012 & 0.009 & 0.008 & 0.007 & 0.006 & 0.006 \\
& $35^{\circ}$ & 0.015 & 0.013 & 0.012 & 0.009 & 0.008 & 0.007 & 0.006 & 0.006 \\
& $90^{\circ}$ & 0.015 & 0.013 & 0.012 & 0.009 & 0.008 & 0.006 & 0.005 & 0.005 \\
& $120^{\circ}$ & 0.015 & 0.013 & 0.012 & 0.009 & 0.008 & 0.007 & 0.006 & 0.006 \\
\hline \hline & FOV & $6^{\circ}$ & $10^{\circ}$ & $20^{\circ}$ & $35^{\circ}$ & $90^{\circ}$ & $120^{\circ}$ & & \\
Vision only (m): & & 0.011 & 0.018 & 0.035 & 0.064 & 0.205 & 0.345 & & \\
\hline
\end{tabular}

Table 7: Standard deviation $(\mathrm{m})$ of recovered $\Delta x$ when ambiguity recovery is successful, $\sigma_{\phi}=0.0115$

\begin{tabular}{||c|cccccccc||}
\hline GNSS-only/GNSS-vision (s) & & & & & & & & \\
Satellites (\#): & 2 & 3 & 4 & 5 & 6 & 10 & 15 & 20 \\
\hline$\sigma_{\phi}(m)$ & & & & & & & & \\
0.001 & $-/ 5.9$ & $-/ 1.1$ & $149.4 / 1.0$ & $77.6 / 1.0$ & $15.6 / 1.0$ & $1.0 / 1.0$ & $1.0 / 1.0$ & $1.0 / 1.0$ \\
0.003 & $-/ 6.1$ & $-/ 1.1$ & $149.4 / 1.0$ & $81.1 / 1.0$ & $36.1 / 1.0$ & $1.4 / 1.0$ & $1.0 / 1.0$ & $1.0 / 1.0$ \\
0.005 & $-/ 5.9$ & $-/ 1.1$ & $149.7 / 1.0$ & $89.1 / 1.0$ & $40.4 / 1.0$ & $2.2 / 1.0$ & $1.0 / 1.0$ & $1.0 / 1.0$ \\
0.0075 & $-/ 6.9$ & $-/ 2.1$ & $149.7 / 1.0$ & $91.1 / 1.0$ & $69.8 / 1.0$ & $3.7 / 1.0$ & $1.3 / 1.0$ & $1.0 / 1.0$ \\
0.010 & $-/ 6.1$ & $-/ 3.6$ & $149.7 / 1.1$ & $130.1 / 1.1$ & $83.8 / 1.0$ & $5.4 / 1.0$ & $1.6 / 1.0$ & $1.0 / 1.0$ \\
0.0125 & $-/ 6.6$ & $-/ 4.9$ & $149.7 / 1.3$ & $139.7 / 1.2$ & $63.0 / 1.0$ & $7.6 / 1.0$ & $2.1 / 1.0$ & $1.0 / 1.0$ \\
0.015 & $-/ 6.6$ & $-/ 4.9$ & $149.8 / 1.7$ & $142.7 / 1.6$ & $73.1 / 1.3$ & $9.3 / 1.1$ & $2.8 / 1.0$ & $1.7 / 1.0$ \\
0.020 & $-/ 6.6$ & $-/ 5.6$ & $150.5 / 2.1$ & $144.5 / 2.0$ & $100.0 / 1.8$ & $11.6 / 1.2$ & $4.0 / 1.1$ & $2.5 / 1.1$ \\
0.025 & $-/ 6.7$ & $-/ 6.5$ & $150.3 / 2.4$ & $148.5 / 2.2$ & $97.3 / 2.2$ & $15.0 / 1.4$ & $5.7 / 1.4$ & $3.3 / 1.3$ \\
0.030 & $-/ 8.3$ & $-/ 6.4$ & $150.7 / 3.8$ & $148.1 / 3.2$ & $106.4 / 2.7$ & $23.3 / 1.9$ & $8.1 / 1.8$ & $4.7 / 1.8$ \\
0.035 & $-/ 8.1$ & $-/ 6.0$ & $151.4 / 3.9$ & $147.5 / 3.5$ & $107.9 / 3.3$ & $17.7 / 2.2$ & $8.7 / 2.2$ & $5.4 / 2.2$ \\
\hline
\end{tabular}

Table 8: Average time (s) to recover double differenced integer ambiguity, $\mathrm{FOV}=90^{\circ}$
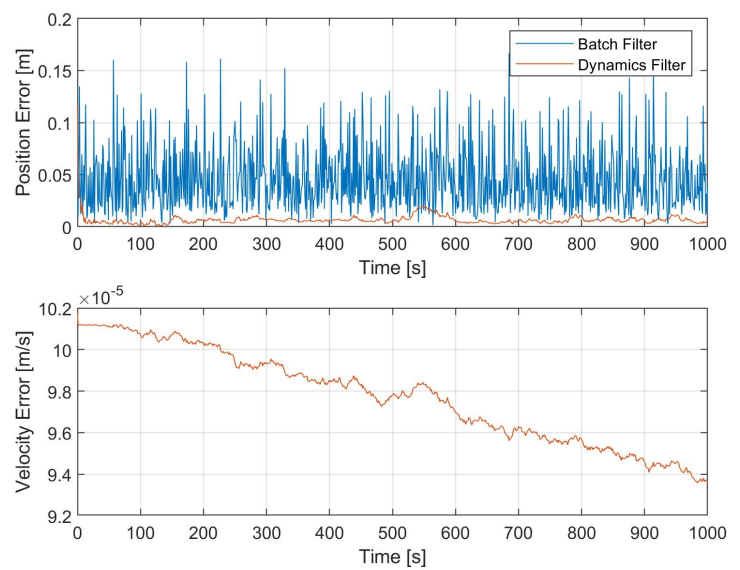

Figure 13: GNSS only error.
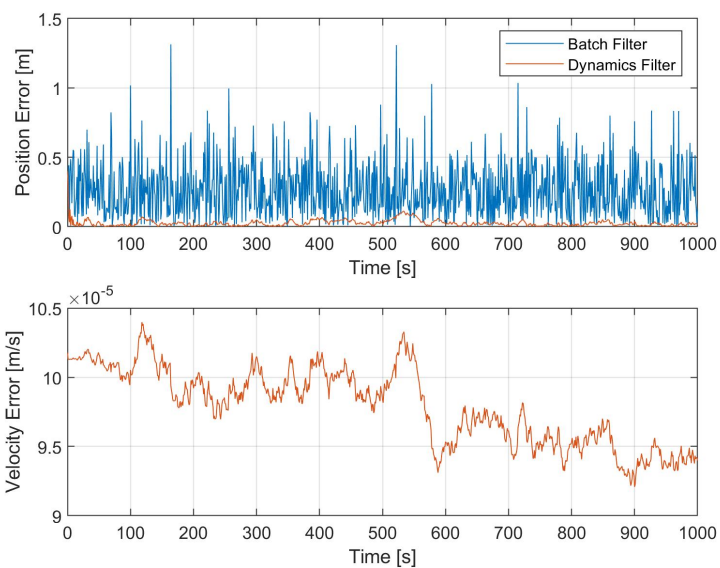

Figure 14: Vision only error.

characterized by weak signals and high GDOP and in presence of strong multipath. The fusion of monocular images with the CDGNSS observations in a tight integration was used to achieve higher relative positioning accuracy, better robustness, and better continuity when navigating in space above the GNSS constellation. The vision-based estimates are exploited to aid to the ambiguity resolution and to increase the accuracy achievable when using carrier-phase tracking measurements. A precise ambiguity recovery is obtained even when fewer than 4 satellites maintain phase lock, when only GNSS would otherwise fail. At the same time the integrated DGNSS-Vision solution is also more accurate 

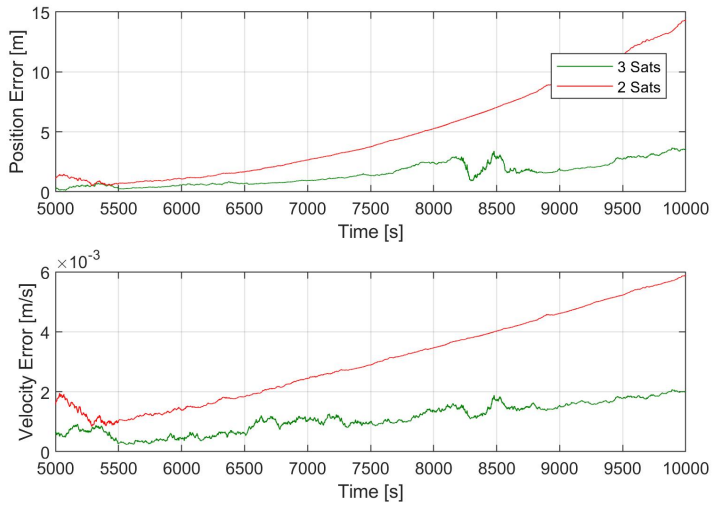

Figure 15: Absolute error with reduced visibility.
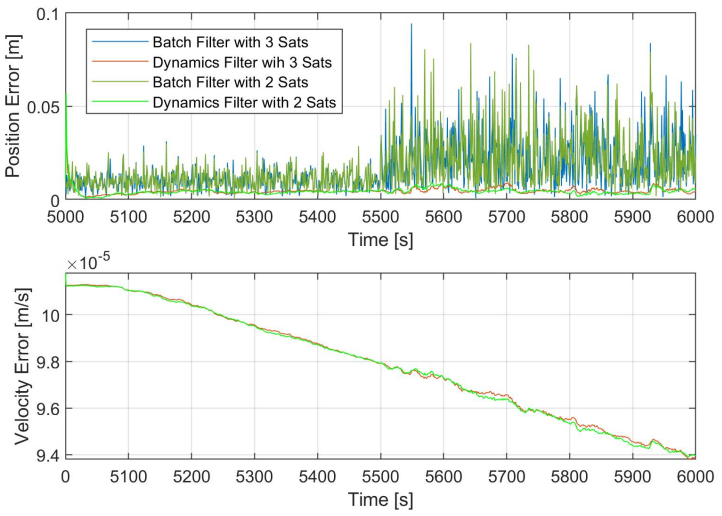

Figure 16: Relative error with reduced visibility.

and robust than the vision only solution, which is strongly affected by the distance of the target, FOV and illumination conditions.

The proposed method was validated with numerical simulations, using a dataset of images synthetically generated according to a chaser/target relative motion in Geostationary Earth Orbit (GEO), and realistic carrier phase and code GNSS observations simulated at the receiver position in the same orbit, taking into account all different GNSS signal delay sources as well as their availability based on their carrier-to-noise-ratio and receiver sensitivity. It is shown that an accuracy better than $2 \mathrm{~mm}$ and $0.01 \mathrm{~mm} / \mathrm{s}$ is achieved with the proposed solution refined with a relative dynamics filter. The achieved accuracy is better than the accuracy achievable when using DGNSS or vision only for large relative distances, different FOVs, low GNSS signal-to-noise ratios, and high GDOP values.

In the future, higher orbits up to the Moon will be considered. These orbits are characterized by weaker signals, reduced satellite availability, and larger GDOP, so the benefits resulting from the fusion should be greater. Due to maintained stability with a large FOV image and fast ambiguity recovery, this approach would be an ideal candidate for formation flying and autonomous swarm applications where the risk of collision is much higher and there may not be the time to wait for a solution convergence. With enough cooperative receivers, the factor graph formulation could be used to decentralize the problem as has been done in some SLAM applications.

This algorithm also suggests the possibility of fusing higher frequency optical measurements with higher carrier phase sampling rates. When vision is present, the code based pseudorange measurements are not strictly necessary. A higher sampling frequency would potentially allow sub-second ambiguity recovery. This approach will be investigated. Finally the dynamics filter will be fused into the batch estimator for a more robust and accurate solution.

\section{ACKNOWLEDGMENTS}

The first author was supported by the Swiss National Science Foundation (SNSF). This work was also supported in part by the Jet Propulsion Laboratory (JPL). Government sponsorship is acknowledged. The authors thank F. Y. Hadaegh, A. Rahmani, and S. R. Alimo.

\section{References}

[1] AuraCAD. https://nasa3d.arc.nasa.gov/detail/aura-eoe3d. Accessed: 2019-06-25.

[2] AuraCAD. https://grabcad.com/library/cubesat-u2-1. Accessed: 2019-06-25.

[3] Blender. https://www.blender.org. Accessed: 2019-06-25.

[4] Satbeams. https://www.satbeams.com/satellites?id=2223. Accessed: 2019-06-25.

[5] STK. http://help.agi.com/stk/index.htmhpop/hpop.htm. Accessed: 2019-06-25. 
[6] ICD-GPS-705C navstar GPS space segment/user segment L5 inter-faces. 2012.

[7] ICD-GPS-200H navstar GPS space segment / user segment interfaces. 2013.

[8] V. Barreau, B. Priot, M. Sahmoudi, and V. Calmettes. A new approach for deep integration of GNSS and visionaided MEMS IMU. In Proceedings of the 23rd International Technical Meeting of The Satellite Division of the Institute of Navigation, pages 464-475, 2001.

[9] P. Blunt, C. Botteron, V. Capuano, and S. Ghamari. Ultra-high sensitivity state-of-the-art receiver for space applications. In 2016 8th ESA Workshop on Satellite Navigation Technologies and European Workshop on GNSS Signals and Signal Processing (NAVITEC), Dec 2016.

[10] V. Capuano. GNSS-based navigation for lunar missions. Technical report, EPFL, 2016.

[11] V. Capuano, P. Blunt, C. Botteron, and P. Farine. Orbital filter aiding of a high sensitivity GPS receiver for lunar missions. Navigation: Journal of The Institute of Navigation, 64(3):323-338, 2017.

[12] V. Capuano, C. Botteron, and P. Farine. GNSS performances for MEO, GEO and HEO. SPACE COMMUNICATIONS AND NAVIGATION SYMPOSIUM - Space-Based Navigation Systems and Services, 2013.

[13] V. Capuano, C. Botteron, J. Leclère, J. Tian, Y. Wang, and P. Farine. Feasibility study of GNSS as navigation system to reach the moon. Acta Astronautica, 116:186-201, 2015.

[14] V. Capuano, K. Kim, J. Hu, A. Harvard, and S. Chung. Monocular-based pose determination of uncooperative known and unknown space objects. In 69th International Astronautical Congress (IAC), Oct 2018.

[15] V. Capuano, R. Opromolla, G. Cuciniello, V. Pesce, S. Sarno, G. Capuano, M. Lavagna, M. Grassi, F. Corraro, P. Tabacco, et al. A highly integrated navigation unit for on-orbit servicing missions. In 69th International Astronautical Congress (IAC 2018), pages 1-13, 2018.

[16] V. Capuano, E. Shehaj, P. Blunt, C. Botteron, and P. Farine. An adaptive GNSS-based reduced dynamic approach for real time autonomous navigation from the earth to the moon. Pacific PNT 2017, 2017.

[17] V. Capuano, E. Shehaj, P. Blunt, C. Botteron, and P. Farine. High accuracy GNSS based navigation in GEO. Acta Astronautica, 136:332-341, 2017.

[18] V. Capuano, E. Shehaj, C. Botteron, P. Blunt, P. Farine, and B. Wang. Availability and ranging error analysis for a GPS L1/L5 receiver navigating to the moon. In 2017 European Navigation Conference (ENC), pages 348-358. Ieee, 2017.

[19] X. Chang, X. Yang, and T. Zhou. Mlambda: a modified lambda method for integer least-squares estimation. Journal of Geodesy, 79(9):552-565, 2005.

[20] P. De Jonge, C. Tiberius, et al. The LAMBDA Methods for Integer Ambiguity Estimation: Implementation Aspects, volume 12. Verlag der Delft Univers. of Technolog., 1996.

[21] F. Dellaert, M. Kaess, et al. Factor graphs for robot perception. Foundations and Trends $\AA$ in Robotics, 6(1-2):1$139,2017$.

[22] S. D'Amico and O. Montenbruck. Differential GPS: An enabling technology for formation flying satellites. In Small Satellite Missions for Earth Observation, pages 457-465. Springer, 2010.

[23] U. Engel. Improving position accuracy by combined processing of Galileo and GPS satellite signals. In 2008 11th International Conference on Information Fusion, pages 1-8. IEEE, 2008.

[24] P. Gurfil. Relative motion between elliptic orbits: generalized boundedness conditions and optimal formationkeeping. Journal of Guidance, Control, and Dynamics, 28(4):761-767, 2005.

[25] S. U. Hwu and Y. C. Loh. Space station GPS multipath analysis and validation. In 1999 IEEE 49th Vehicular Technology Conference (Cat. No. 99CH36363), volume 1, pages 757-761. IEEE, 1999. 
[26] M. Kaess, H. Johannsson, R. Roberts, V. Ila, J. J. Leonard, and F. Dellaert. iSAM2: Incremental smoothing and mapping using the bayes tree. The International Journal of Robotics Research, 31(2):216-235, 2012.

[27] R. E. Kalman. A new approach to linear filtering and prediction problems. ASME Journal of Basic Engineering, 1960.

[28] E. Kaplan and C. Hegarty. Understanding GPS: principles and applications. Artech house, 2005.

[29] R. Kroes. Precise relative positioning of formation flying spacecraft using GPS. 2006.

[30] S. Lee. On-the-fly carrier phase ambiguity resolution without using pseudorange measurements for satellitebased differential positioning. PhD thesis, Ohio University, 1994.

[31] P. Magnusson. Antenna for GNSS reception in geo-orbit, 2014.

[32] S. Mohiuddin and M. L. Psiaki. High-altitude satellite relative navigation using carrier-phase differential global positioning system techniques. Journal of Guidance, Control, and Dynamics, 30(5):1427-1436, 2007.

[33] A. Myronenko and X. Song. Point set registration: Coherent point drift. IEEE Transaction on Pattern Analysis and Machine Intelligence, 32(12):2262-2275, 2010.

[34] K. M. Pesyna Jr et al. Advanced techniques for centimeter-accurate GNSS positioning on low-cost mobile platforms. $\mathrm{PhD}$ thesis, 2015.

[35] B. Priot, C. Peillon, V. Calmettes, and M. Sahmoudi. Performance assessment of an ultra-tightly coupled visionaided INS/GNSS navigation system. In Proceedings of the 2011 International Technical Meeting of The Institute of Navigation, 2011.

[36] M. L. Psiaki and S. Mohiuddin. Relative navigation of high-altitude spacecraft using dual-frequency civilian CDGPS. Earth, 3:7, 2005.

[37] M. L. Psiaki and S. Mohiuddin. Modeling, analysis, and simulation of GPS carrier phase for spacecraft relative navigation. Journal of Guidance, Control, and Dynamics, 30(6):1628-1639, 2007.

[38] A. Reichert, T. Meehan, and T. Munson. Toward decimeter-level real-time orbit determination: a demonstration using the SAC-C and CHAMP spacecraft. Proceediings of the ION-GPS-2002, pages 24-27, 2002.

[39] S. Segal and P. Gurfil. Effect of kinematic rotation-translation coupling on relative spacecraft translational dynamics. Journal of Guidance, Control, and Dynamics, 32(3):1045-1050, 2009.

[40] E. Shehaj, V. Capuano, C. Botteron, P. Blunt, and P. Farine. GPS based navigation performance analysis within and beyond the space service volume for different transmitters' antenna patterns. Aerospace, 4(3), 2017.

[41] D. Simon. Optimal state estimation: Kalman, H infinity, and nonlinear approaches. John Wiley \& Sons, 2006.

[42] P. J. G. Teunissen. Theory of carrier phase ambiguity resolution. Wuhan University Journal of Natural Sciences, 8(2):471, 2003.

[43] D. H. Won, E. Lee, M. Heo, S. Sung, J. Lee, and Y. J. Lee. GNSS integration with vision-based navigation for low GNSS visibility conditions. GPS solutions, 18(2):177-187, 2014.

[44] S. Wu and Y. E. Bar-Sever. Real-time sub-cm differential orbit determination of two low-earth orbiters with GPS bias fixing. 2006.

[45] Y. Wu and Z. Hu. PnP problem revisited. Journal of Mathematical Imaging and Vision, 24(1):131-141, December 2005 .

[46] K. Yamanaka and F. Ankersen. New state transition matrix for relative motion on an arbitrary elliptical orbit. Journal of guidance, control, and dynamics, 25(1):60-66, 2002. 